\title{
RATIONAL HOMOLOGY 5-SPHERES WITH POSITIVE RICCI CURVATURE
}

\author{
Charles P. Boyer and Krzysztof Galicki
}

\begin{abstract}
We prove that for every integer $k>1$ there is a simply connected rational homology 5 -sphere $M_{k}^{5}$ with spin such that $H_{2}\left(M_{k}^{5}, \mathbb{Z}\right)$ has order $k^{2}$, and $M_{k}^{5}$ admits a Riemannian metric of positive Ricci curvature. Moreover, if the prime number decomposition of $k$ has the form $k=p_{1} \cdots p_{r}$ for distinct primes $p_{i}$ then $M_{k}^{5}$ is uniquely determined.
\end{abstract}

\section{Introduction}

Recently, Stephan Stolz brought to our attention the fact that preciously little is known about the existence of metrics of positive Ricci curvature on simply connected 5-manifolds in the presence of torsion in $H_{2}(M, \mathbb{Z})$. Indeed up until now there appears to be only one known non-trivial example of a rational homology 5-sphere admitting a metric of positive Ricci curvature. This is somewhat surprising in light of the fact that it is precisely in dimension 5 that there is a diffeomorphism classification of closed oriented simply connected 5-manifolds [Bar, Sm].

Regarding the question of positive Ricci curvature on such manifolds, there is the well-known result of Sha and Yang [SY] which, in dimension 5, implies that any $k$-fold connected sum of $S^{2} \times S^{3}$ admits a metric of positive Ricci curvature (an alternative proof using methods similar to this note was given in [BGN2]). The non-trivial $S^{3}$ bundle over $S^{2}$ admits a metric of positive Ricci curvature because of its bundle structure [Na]. Lastly, we have the two symmetric spaces $S^{5}$ and $S U(3) / S O(3)$ which are actually Einstein [Bes]. The last example is the rational homology 5 -sphere mentioned above. It is non-spin with $H_{2}(M, \mathbb{Z})=\mathbb{Z}_{2}$. In this note we prove the following.

Theorem A. For every integer $k>1$, there exists a simply connected rational homology 5-sphere $M_{k}^{5}$ such that $H_{2}\left(M_{k}^{5}, \mathbb{Z}\right)$ has order $k^{2}, w_{2}\left(M_{k}^{5}\right)=0$, and $M_{k}^{5}$ admits a Sasakian metric with positive Ricci curvature.

Our result establishes the existence of metrics with positive Ricci curvature on an infinite number of rational homology 5 -spheres, but not all the rational homology 5-spheres in Smale's classification of simply connected closed 5manifolds with spin, that is vanishing second Stiefel-Whitney class, $w_{2}(M)$. It

Received April 25, 2002.

During the preparation of this work the authors were partially supported by NSF grants DMS-9970904 and DMS-0203219. 
is worth mentioning that our examples are complementary to the homogeneous manifold $S U(3) / S O(3)$ whose second and third Stiefel-Whitney classes are both non-vanishing, and thus admits neither a spin structure nor an almost contact structure. In fact our methods use contact Riemannian geometry, and a result of Geiges [Gei] says that any simply connected rational homology 5-sphere that admits a contact structure must be spin. Recall that by Smale's theorem [Sm] any rational homology 5 -sphere $M^{5}$ with $w_{2}(M)=0$ can be written uniquely as (for our purposes it is more convenient to rephrase Smale's result in terms of elementary divisors instead of invariant factors as he does):

$$
M^{5}=M_{p_{1}^{s_{1}}}^{5} \# \cdots \# M_{p_{r}^{s_{r}}}^{5}
$$

for some positive integers $r, s_{1}, \cdots, s_{r}$ where the $p_{i}$ 's are (not necessarily distinct) primes, and $H_{2}\left(M_{p_{i}^{s_{i}}}^{5}, \mathbb{Z}\right)=\mathbb{Z}_{p_{i}^{s_{i}}} \oplus \mathbb{Z}_{p_{i}^{s_{i}}}$. In particular, the order of $H_{2}\left(M^{5}, \mathbb{Z}\right)$ is always a perfect square.

Our construction gives all possible orders of $\mathrm{H}_{2}$, but it does not pin down the group precisely in all cases. However, the form of $H_{2}$ given by Smale's theorem says that the elementary divisors of $H_{2}$ must occur in pairs. Thus, when all of the primes $p_{i}$ in equation (1) are distinct and all of the $s_{i}$ 's equal 1 , the order of $H_{2}$ uniquely determines the manifold. For example, if $\left|H_{2}\left(M^{5}, \mathbb{Z}\right)\right|=36$, the elementary divisors must be $\{2,2,3,3\}$. This determines $M^{5}$ to be $M_{2}^{5} \# M_{3}^{5}$. However, if for example, $\left|H_{2}\left(M^{5}, \mathbb{Z}\right)\right|=64$ the elementary divisors can be $\left\{2^{3}, 2^{3}\right\},\left\{2,2,2^{2}, 2^{2}\right\}$, or $\{2,2,2,2,2,2\}$, giving the possibilities for $M^{5}$ as $M_{2^{3}}^{5}, M_{2}^{5} \# M_{2^{2}}^{5}$, or $M_{2}^{5} \# M_{2}^{5} \# M_{2}^{5}$. In this case we are unable to determine which manifolds occur. Thus, we stop short of proving that all simply connected rational homology 5-spheres with $w_{2}(M)=0$ admit metrics with positive Ricci curvature, although we certainly believe this to be the case. However, in cases when the order determines the group we have the following corollary.

Corollary B. For every positive integer $r$ and every list of distinct primes $p_{1}, \cdots, p_{r}$, the manifolds

$$
M^{5}=M_{p_{1}}^{5} \# \cdots \# M_{p_{r}}^{5}
$$

admits Sasakian metrics with positive Ricci curvature.

In the absence of known obstructions the question regarding the existence of positive Ricci curvature metrics on any closed simply connected 5-manifold is an intriguing one. However, in view of Geiges' result mentioned above, the answer to the general question is beyond the scope of our current techniques. On the other hand, we believe that in the spin case one should be able to extend Theorem A to many other cases and possibly obtain a complete classification. First it is reasonable that actually all spin rational homology 5 -spheres can be realized as links with positive Sasakian structure. The main problem in proving this is in computing the torsion group, for which one needs to work, not just over $\mathbb{C}$, but with the integral monodromy, and this is known to be difficult. There is a conjectured algorithm due to Orlik [Or3] which has been verified in certain special cases, and we plan to address this question in the future. Another 
approach would be to use surgery, assuming that one has the existence of positive Ricci curvature metrics on the indecomposable pieces $M_{k}$. However, apparently the only known method, e.g. [SY], entails finding 2-spheres in $M_{k}$ such that the metric in a neighborhood of the $S^{2}$ is in standard form [St], and this appears to be obstructed by the 2-torsion.

\section{Positive Sasakian Geometry}

Let $(M, J)$ is a compact complex manifold and $g$ a Kähler metric on $M$, with Kähler form M. Suppose that $\rho^{\prime}$ is a real, closed $(1,1)$-form on $M$ with $\left[\rho^{\prime}\right]=2 \pi c_{1}(M)$. Then there exists a unique Kähler metric $g^{\prime}$ on $M$ with Kähler form $\omega^{\prime}$, such that $[\omega]=\left[\omega^{\prime}\right] \in H^{2}(M, \mathbb{R})$, and the Ricci form of $g^{\prime}$ is $\rho^{\prime}$. The above statement is the celebrated Calabi Conjecture which was posed by Eugene Calabi in 1954. The conjecture in its full generality was eventually proved by Yau in 1976. In the Fano case when $c_{1}(M)>0$, i.e., when the first Chern class can be represented by a positive-definite real, closed $(1,1)$-form $\rho^{\prime}$ on $M$, the conjecture implies that the Kähler form of $M$ can be represented by a metric of positive Ricci curvature. The key idea behind the proof of Theorem A is based on a more general Calabi Problem when $M$ is not necessarily a smooth manifold but rather a $V$-manifold or an orbifold [DK, Joy]. In the context of foliations one actually can prove a "transverse Yau theorem" and this was done by $\mathrm{El}$ Kacimi-Alaoui in 1990 [ElK]. In [BGN2] we adapted this to a very special case of Sasakian foliations.

Recall [Bl, YK] that a Sasakian structure on a manifold $M$ of dimension $2 n+1$ is a metric contact structure $(\xi, \eta, \Phi, g)$ such that the Reeb vector field $\xi$ is a Killing field and whose underlying almost CR structure is integrable. Briefly, let $(M, \mathcal{D})$ be a contact manifold, and choose a 1 -form $\eta$ so that $\eta \wedge(d \eta)^{n} \neq 0$ and $\mathcal{D}=\operatorname{ker} \eta$. The pair $(\mathcal{D}, \omega)$, where $\omega$ is the restriction of $d \eta$ to $\mathcal{D}$ gives $\mathcal{D}$ the structure of a symplectic vector bundle. Choose an almost complex structure $J$ on $\mathcal{D}$ that is compatible with $\omega$, that is $J$ is a smooth section of the endomorphism bundle End $\mathcal{D}$ that satisfies

$$
J^{2}=-\mathbb{I}, \quad d \eta(J X, J Y)=d \eta(X, Y), \quad d \eta(X, J X)>0
$$

for any smooth sections $X, Y$ of $\mathcal{D}$. Notice that $J$ defines a Riemannian metric $g_{\mathcal{D}}$ on $\mathcal{D}$ by setting $g_{\mathcal{D}}(X, Y)=d \eta(X, J Y)$. One easily checks that $g_{\mathcal{D}}$ satisfies the compatibility condition $g_{\mathcal{D}}(J X, J Y)=g_{\mathcal{D}}(X, Y)$. Now we can extend $J$ to an endomorphism $\Phi$ on all of $T M$ by putting $\Phi=J$ on $\mathcal{D}$ and $\Phi \xi=0$. Likewise we can extend the metric $g_{\mathcal{D}}$ on $\mathcal{D}$ to a Riemannian metric $g$ on $M$ by setting

$$
g=g_{\mathcal{D}}+\eta \otimes \eta
$$

The quadruple $(\xi, \eta, \Phi, g)$ is called a metric contact structure on $M$. If in addition $\xi$ is a Killing vector field and the almost complex structure $J$ on $\mathcal{D}$ is integrable the underlying almost contact structure is said to be normal and $(\xi, \eta, \Phi, g)$ is called a Sasakian structure. The fiduciary examples of compact Sasakian 
manifolds are the odd dimensional spheres $S^{2 n+1}$ with the standard contact structure and standard round metric $g$.

Every Sasakian structure $\mathcal{S}=(\xi, \eta, \Phi, g)$ has a 1-dimensional foliation $\mathcal{F}_{\xi}$ associated to it, defined by the flow of the Reeb vector field $\xi$ and called the characteristic foliation. Associated with this foliation are important invariants, namely, the basic cohomology groups $H_{B}^{p}\left(\mathcal{F}_{\xi}\right)$, (cf. [Ton]) and in particular one can consider the basic first Chern class $c_{1}\left(\mathcal{F}_{\xi}\right)$ [ElK, BGN2] as an element in $H_{B}^{2}\left(\mathcal{F}_{\xi}\right)$. These are not only invariants of the Sasakian structure, but of the entire deformation class of Sasakian structures. Notice that on a compact Sasakian manifold $H_{B}^{2}\left(\mathcal{F}_{\xi}\right) \neq 0$ since $[d \eta]_{B}$ is a non-vanishing class.

Definition 1.3. A Sasakian manifold $M$ is said to be positive if its basic first Chern class $c_{1}\left(\mathcal{F}_{\xi}\right)$ can be represented by a basic positive definite $(1,1)$-form.

As in [BGN2, BGN4] we consider deformation classes $\mathfrak{F}\left(\mathcal{F}_{\xi}\right)$ of Sasakian structures that have the same characteristic foliation. Recall that two Sasakian structures $\mathcal{S}=(\xi, \eta, \Phi, g)$ and $\mathcal{S}^{\prime}=\left(\xi^{\prime}, \eta^{\prime}, \Phi^{\prime}, g^{\prime}\right)$ in $\mathfrak{F}\left(\mathcal{F}_{\xi}\right)$ on a smooth manifold $M$ are said to be a-homologous if there is an $a \in \mathbb{R}^{+}$such that $\xi^{\prime}=a^{-1} \xi$ and $\left[d \eta^{\prime}\right]_{B}=a[d \eta]_{B}$. On a rational homology sphere every $\mathcal{S} \in \mathfrak{F}\left(\mathcal{F}_{\xi}\right)$ belongs to precisely one of two $a$-homology classes corresponding to a given Sasakian structure or its conjugate. In [BGN2] we proved, using El-Kacimi-Alaoui's [ElK] "transverse Yau Theorem":

Theorem 1.4. [BGN2] Let $\mathcal{S}=(\xi, \eta, \Phi, g)$ be a positive Sasakian structure on a compact manifold $M$ of dimension $2 n+1$. Then $M$ admits a Sasakian structure $\mathcal{S}^{\prime}=\left(\xi^{\prime}, \eta^{\prime}, \Phi^{\prime}, g^{\prime}\right)$ with positive Ricci curvature a-homologous to $\mathcal{S}$ for some $a>0$.

Theorem 1.4 says that to prove the existence of a Sasakian metric with positive Ricci curvature it suffices to prove the existence of positive Sasakian structures. In the next section we shall discuss how to construct positive Sasakian structures on homotopy 5-spheres, and prove Theorem A of the Introduction.

\section{The Construction}

Our 5-manifolds are constructed as $k$-fold branched covers of $S^{5}$ branched over certain Seifert manifolds that are in turn $S^{1}$ orbifold V-bundles over a compact Riemann surface of genus $g$. Our construction is similar to that in [Sav]. Let $f_{3}\left(z_{1}, z_{2}, z_{3}\right)$ be a weighted homogeneous polynomial of an isolated hypersurface singularity in $\mathbb{C}^{3}$ with weights $\mathbf{w}=\left(w_{1}, w_{2}, w_{3}\right)$ and degree $d$. The link $L_{\mathbf{w}}$ defined by $L_{\mathbf{w}}=\left\{f_{3}=0\right\} \cap S^{5}$ is a Seifert fibration over an algebraic curve $C_{\mathbf{w}}$ in the weighted projective space $\mathbb{P}(\mathbf{w})$. Let $g=g(\mathbf{w})$ denote the genus of the curve $C_{\mathbf{w}}$. Then,

Proposition 2.1. Let $L_{f}$ denote the link of the weighted homogeneous polynomial

$$
f=z_{0}^{k}+f_{3}\left(z_{1}, z_{2}, z_{3}\right)
$$


with weights $(d, k \mathbf{w})$ where $k$ is an integer $>1$ where $f_{3}$ is a weighted homogeneous polynomial of degree $d$ with weights $\mathbf{w}=\left(w_{1}, w_{2}, w_{3}\right)$ as above. Suppose further that $\operatorname{gcd}(d, k)=1$. Then the link $L_{f}$ is a smooth simply connected rational homology 5-sphere such that the order of $H_{2}\left(L_{f}, \mathbb{Z}\right)$ is $k^{2 g}$. Furthermore, $L_{f}$ admits Sasakian metrics with positive Ricci curvature.

Proof. Let us briefly recall the construction of the Alexander (characteristic) polynomial $\Delta_{3}(t)$ in [MO] associated to a 3-dimensional link $L_{f_{3}}$. It is the characteristic polynomial of the monodromy map $\mathbb{I}-h_{*}: H_{2}(F, \mathbb{Z}) \longrightarrow H_{2}(F, \mathbb{Z})$ induced by the $S_{\mathbf{w}}^{1}$ action on the Milnor fibre $F$. Thus, $\Delta_{3}(t)=\operatorname{det}\left(t \mathbb{I}-h_{*}\right)$. Now both $F$ and its closure $\bar{F}$ are homotopy equivalent to a bouquet of 3 -spheres, and the boundary of $\bar{F}$ is the link $L_{f_{3}}$. Now $L_{f_{3}}$ is connected and its Betti numbers $b_{1}\left(L_{f_{3}}\right)=b_{2}\left(L_{f_{3}}\right)$ equal the number of factors of $(t-1)$ in $\Delta_{3}(t)$. Now since the curve $C_{\mathbf{w}}$ is algebraic, $b_{1}\left(L_{f_{3}}\right)=2 g$ where $g$ is the genus of $C_{\mathbf{w}}$. Following Milnor and Orlik we let $\Lambda_{j}$ denote the divisor of $t^{j}-1$ in the group ring $\mathbb{Z}\left[\mathbb{C}^{*}\right]$. Then the divisor of $\Delta_{3}(t)$ is given by

$$
\operatorname{div} \Delta_{3}=\prod_{i=1}^{3}\left(\frac{\Lambda_{u_{i}}}{v_{i}}-1\right)
$$

where we write $\frac{d}{w_{i}}=\frac{u_{i}}{v_{i}}$ in irreducible form. Using the relations $\Lambda_{a} \Lambda_{b}=$ $\operatorname{gcd}(a, b) \Lambda_{l c m(a, b)}$, equation 2.2 takes the form $\sum a_{j} \Lambda_{j}-1$, where $a_{j} \in \mathbb{Z}$ and the sum is taken over the set of all least common multiples of all combinations of the $u_{1}, \cdots, u_{n}$. The Alexander polynomial is then given by

$$
\Delta_{3}(t)=(t-1)^{-1} \prod_{j}\left(t^{j}-1\right)^{a_{j}}
$$

and

$$
b_{1}\left(L_{f_{3}}\right)=2 g=\sum_{j} a_{j}-1 .
$$

Now we compute the divisor div $\Delta_{4}$ of the Alexander polynomial $\Delta_{4}(t)$ for $f$. We have

$$
\begin{gathered}
\operatorname{div} \Delta_{4}=\left(\Lambda_{k}-1\right) \operatorname{div} \Delta_{3}=\left(\Lambda_{k}-1\right)\left(\sum a_{j} \Lambda_{j}-1\right) \\
=\sum_{j} \operatorname{gcd}(k, j) a_{j} \Lambda_{\operatorname{lcm}(k, j)}-\sum_{j} a_{j} \Lambda_{j}-\Lambda_{k}+1 .
\end{gathered}
$$

Since the $j$ 's run through all the least common multiples of the set $\left\{u_{1}, \cdots, u_{n}\right\}$ and $\operatorname{gcd}\left(k, u_{i}\right)=1$ for all $i$, we see that for all $j, \operatorname{gcd}(k, j)=1$. This implies

$$
b_{2}\left(L_{f}\right)=\sum_{j} a_{j}-\sum_{j} a_{j}-1+1=0
$$

Thus, $L_{f}$ is a rational homology sphere. Next we compute the Alexander polynomial for $L_{f}$.

$$
\Delta_{4}(t)=\frac{(t-1)}{\left(t^{k}-1\right)} \prod_{j} \frac{\left(t^{k j}-1\right)^{a_{j}}}{\left(t^{j}-1\right)^{a_{j}}}
$$




$$
=\left(t^{k-1}+\cdots+t+1\right)^{-1} \prod_{j}\left(\frac{t^{k j-1}+\cdots+t+1}{t^{j-1}+\cdots+t+1}\right)^{a_{j}} .
$$

This gives

$$
\Delta_{4}(1)=k^{-1} \prod_{j}\left(\frac{k j}{j}\right)^{a_{j}}=k^{\Sigma_{j} a_{j}-1}=k^{2 g} .
$$

So by $[\mathrm{MO}]$ the order of $H_{2}\left(L_{f}, \mathbb{Z}\right)$ is $\Delta_{4}(1)=k^{2 g}$.

To finish the proof it suffices by Theorem 1.4 to show that the induced Sasakian structure on $L_{f}$ is positive, i.e. that the basic first Chern class $c_{1}\left(\mathcal{F}_{\xi}\right) \in$ $H_{B}^{2}\left(\mathcal{F}_{\xi}\right)$ is positive. Now from our previous work [BG1-2,BGN1-4] $L_{f}$ is the total space of a V-bundle over a Kähler orbifold $\mathcal{Z}_{f}$. Moreover, $c_{1}\left(\mathcal{F}_{\xi}\right)$ is just $c_{1}\left(\mathcal{Z}_{f}\right)$ pulled back to $L_{f}$. Thus, it is enough to prove that $\mathcal{Z}_{f}$ is Fano. This follows from Lemma 2.6 below which is a special case of Lemma 3.12 of [BGN4].

Lemma 2.6. As algebraic varieties $\mathcal{Z}_{f}$ is isomorphic to the weighted projective space $\mathbb{P}(\mathbf{w})$. Hence, its Fano index is $|\mathbf{w}|=\sum_{i} w_{i}>0$.

This concludes the proof of Proposition 2.1.

Next we want to show that every order of $H_{2}$ can be realized. First we show that $f_{3}$ realizes curves of any genus. In fact there is a formula due to Orlik and Wagreich $[\mathrm{OW}]$ for the genus of the curve $C_{\mathbf{w}}$ which generalizes the well known genus formula for curves in $\mathbb{P}^{2}$ (See also the books [Dim, Or1]). It is

$$
g\left(C_{\mathbf{w}}\right)=\frac{1}{2}\left(\frac{d^{2}}{w_{1} w_{2} w_{3}}-d \sum_{i<j} \frac{\operatorname{gcd}\left(w_{i}, w_{j}\right)}{w_{i} w_{j}}+\sum_{i} \frac{\operatorname{gcd}\left(d, w_{i}\right)}{w_{i}}-1\right) .
$$

We are interested in the case $g>0$ which implies $w_{1}+w_{2}+w_{3} \leq d$ [Or2]. It is easy to see that there are quasi-smooth weighted homogeneous polynomials $f_{3}$ with arbitrary genus, but we claim that genus one will suffice to realize all rational homology spheres of the form given in our main theorem.

Proposition 2.8. For every integer $k>1$, there exists a rational homology 5 -sphere $M_{k}^{5}$ whose second homology group $H_{2}\left(M_{k}^{5}, \mathbb{Z}\right)$ has order $k^{2}$ and that can be realized as the link $L_{f}$ of a weighted homogeneous polynomial $f$ given in Proposition 2.1 where $f_{3}$ cuts out a projective curve of genus one. Furthermore if $k$ has the form $k=p_{1} \cdots p_{r}$ for distinct primes $p_{i}$, the manifold $M_{k}^{5}$ is uniquely determined up to diffeomorphism.

Proof. By Smale's classification theorem [Sm] and Proposition 2.1 it suffices to exhibit for each integer $k>1$ an infinite family of weighted homogeneous polynomials $f_{3}$ of prime degree $p$ with $g\left(C_{\mathbf{w}}\right)=1$. For then for a given $k$ we can choose $p$ such that $\operatorname{gcd}(k, p)=1$. The infinite family of polynomials is given by

$$
f_{p}\left(z_{1}, z_{2}, z_{3}\right)=z_{1}^{p}+z_{2}^{2} z_{3}+z_{3}^{2} z_{1}
$$


with weights $\mathbf{w}=\left(1, \frac{p+1}{4}, \frac{p-1}{2}\right)$ and degree $p$ where $p$ is a prime of the form $p=4 l-1$. It is well known that there are an infinite number of such primes. The genus formula then gives

$$
\begin{aligned}
g & =\frac{1}{2}\left(\frac{8 p^{2}}{p^{2}-1}-p\left(\frac{4}{p+1}+\frac{2}{p-1}+\frac{8}{p^{2}-1}\right)+1+\frac{4}{p+1}+\frac{2}{p-1}-1\right) \\
& =\frac{1}{2} \frac{1}{p^{2}-1}\left(2 p^{2}-6 p+6 p-2\right)=1,
\end{aligned}
$$

where we have used the fact that $\operatorname{gcd}\left(\frac{p+1}{4}, \frac{p-1}{2}\right)=\operatorname{gcd}(l, 2 l-1)=1$, and this proves the first statement. The second statement follows from the classification of finite Abelian groups and Smale's classification theorem [Sm].

\section{Remarks 2.9.}

(1) In the case that $k=p_{1} \cdots p_{r}$ for distinct primes $p_{i}$, the links cannot be realized using curves of higher genus $(g>1)$.

(2) It is still an open question as to whether all simply connected rational homology 5 -spheres can be realized by our methods, and if so how does one distinguish the different elementary divisors. The curves of higher genus should play a role here.

(3) For each $k>1$ there are an infinite number of $p$ 's that satisfy $\operatorname{gcd}(k, p)=$ 1. This gives rise to an infinite number of Sasakian deformation classes $\mathfrak{F}\left(\mathcal{F}_{\xi}\right)$ each with Sasakian metrics of positive Ricci curvature. It is also quite plausible that the different deformation classes belong to distinct underlying contact structures, but we have not proven this.

\section{Acknowledgments}

The authors would like to thank Stephan Stolz and Wolfgang Ziller for fruitful discussions. The second author would also like to than Max-Planck-Institut in Bonn for hospitality and support.

\section{References}

[Bar] D. Barden, Simply connected five-manifolds, Ann. of Math. (2) 82 (1965), 365-385.

[Bes] A. Besse, Einstein manifolds, Ergebnisse der Mathematik und ihrer Grenzgebiete (3). Springer-Verlag, Berlin, 1987.

[BG1] C. P. Boyer, K. Galicki, On Sasakian-Einstein geometry, Internat. J. Math. 11 (2000), 873-909.

[BG2] _ New Einstein metrics in dimension five, J. Differential Geom. 57 (2001), 443-463.

[BGN1] C.P. Boyer, K. Galicki, M. Nakamaye, On the Geometry of Sasakian-Einstein 5Manifolds, to appear in Math. Ann.; math.DG/0012041

[BGN2] — On positive Sasakian geometry, to appear in Geom. Ded.; math.DG/0104126

[BGN3] _ Einstein metrics on rational homology 7-spheres, to appear in Ann. Inst. Fourier; math.DG/0108113

[BGN4] _ Sasakian geometry, homotopy spheres and positive Ricci curvature, to appear in Topology; math.DG/0201147

[Bl] D. E. Blair, Contact manifolds in Riemannian geometry, Lecture Notes in Mathematics, Vol. 509. Springer-Verlag, Berlin-New York, 1976. 
[DK] J.-P. Demailly, J. Kollár, Semi-continuity of complex singularity exponents and Kähler-Einstein metrics on Fano orbifolds, Ann. Sci. École Norm. Sup. (4) 34 (2001), $525-556$.

[Dim] A. Dimca, Singularities and topology of hypersurfaces, Universitext. Springer-Verlag, New York, 1992.

[ElK] A. El Kacimi-Alaoui, Opérateurs transversalement elliptiques sur un feuilletage riemannien et applications, Compositio Math. 73 (1990), 57-106.

[Gei] H. Geiges, Contact structures on 1-connected 5-manifolds, Mathematika 38 (1991), 303-311.

[Joy] D. Joyce, Compact manifolds with special holonomy, Oxford Mathematical Monographs. Oxford University Press, Oxford, 2000.

[MO] J. Milnor, P. Orlik, Isolated singularities defined by weighted homogeneous polynomials, Topology 9 (1970), 385-393.

[Na] J. Nash, Positive Ricci curvature on fibre bundles, J. Differential Geom. 14 (1979), 241-254.

[Or1] P. Orlik, Seifert manifolds, Lecture Notes in Mathematics, Vol. 291. Springer-Verlag, Berlin-New York, 1972.

[Or2] Weighted homogeneous polynomials and fundamental groups, Topology 9 (1970), 267-273.

[Or3] - On the homology of weighted homogeneous manifolds, Proceedings of the Second Conference on Compact Transformation Groups (Univ. Massachusetts, Amherst, Mass., 1971), Part I, pp. 260-269. Lecture Notes in Math., Vol. 298, Springer, Berlin, 1972.

[OW] P. Orlik and P. Wagreich, Isolated singularities of algebraic surfaces with $\mathbb{C}^{*}$ action, Ann. of Math. (2) 93 (1971), 205-228.

[Sav] I. V. Savel'ev, Structure of singularities of a class of complex hypersurfaces, Mat. Zam. 25 (4) (1979) 497-503; English translation: Math. Notes 25 (1979), no. 3-4, 258-261.

[Sm] S. Smale, On the structure of 5-manifolds, Ann. of Math. (2) 75 (1962), 38-46.

[St] S. Stolz, private communication.

[SY] J.-P. Sha, D.-G Yang, Positive Ricci curvature on the connected sums of $S^{n} \times S^{m}$, J. Differential. Geom. 33 (1991), 127-137.

[Ton] Ph. Tondeur, Geometry of foliations, Monographs in Mathematics, 90. Birkhäuser Verlag, Basel, 1997.

[YK] K. Yano, M. Kon, Structures on manifolds, Series in Pure Mathematics, 3. World Scientific Publishing Co., Singapore, 1984.

Department of Mathematics and Statistics, University of New Mexico, AlbuQUERQUE, NM 87131, U.S.A.

E-mail address: cboyer@math.unm.edu

E-mail address: galicki@math.unm.edu 